\title{
Eduard Mühle: Breslau. Geschichte einer europäischen Metropole. Köln, Weimar, Wien: Böhlau-Verlag 2015, 386 S.
}

DOI: 10.19195/0435-5865.141.42

Breslau wird heute in der Kulturwissenschaft als plurikulturelle Stadt bezeichnet. Nach 1989 erschienen mehrere Buchpublikationen, die das Thema der Breslauer Stadtgeschichte aufgriffen. Als Standardwerk gilt der im Jahre 2000 von Prof. Teresa Kulak verfasste historische Führer Breslaus Wrocław - przewodnik historyczny. ${ }^{1}$ In diesem Buch fasst die Breslauer Historikerin die Vergangenheit der Stadt in einer überschaubaren Form zusammen. Aus den neueren Monographien, die Breslau als einen Kristallisationspunkt der mitteleuropäischen Kultur wahrnehmen, gilt es beispielsweise das 2002 vom britischen Professor Norman Davies und seinem Mitarbeiter Roger Moorhouse geschriebene Buch Breslau. Die Blume Europas. Geschichte einer mitteleuropäischen Stadt zu erwähnen. ${ }^{2}$ Sie vertreten den Standpunkt, Breslau repräsentiere einen „Mikrokosmos“ Mitteleuropas - es befand sich im Schnittpunkt unterschiedlicher kultureller und politischer Einflüsse. Um das zu belegen, führen sie die zahlreichen, in der Stadtgeschichte auftretenden Namen der Odermetropole an. Die Plurikulturalität der Stadt unterstreicht ebenfalls Prof. Andrzej Zawada, ein anerkannter Breslauer Polonist und Literaturkritiker, indem er für das heutige Wrocław einen metaphorischen Namen vorschlägt, der sowohl die polnische als auch die deutsche Vergangenheit berücksichtigt, und zwar: Bresław. ${ }^{3}$ Ausdruck eines großen Interesses an der Odermetropole ist auch ein von Roswitha Schieb verfasster Literarischer Reiseführer. Breslau 4 aus dem Jahre 2004. Überdies wurde im Jahre 2014 eine Monographie Das alte Breslau. Kulturgeschichte einer geistigen Metropole ${ }^{5}$ von Klaus Gerber veröffentlicht. Sein „Buch ist eine Einladung in das alte Breslau, das in seinen geistigen Schätzen nicht anders als in seinen wiedererstanden Bauten eindrucksvoll in der Gegenwart fortlebt. Die Wahl zur Europäischen Kulturhauptstadt 2016 stellt dies sinnfällig unter Beweis". 6

Die Tendenz, Breslau als ein außergewöhnliches Phänomen Mitteleuropas zu präsentieren, wird auch von Eduard Mühle fortgesetzt, einem angesehenen deutschen Historiker, seit 2005 Professor für Geschichte Ostmitteleuropas, der 2008-2013 das Deutsche Historische Institut in Warschau leitete. In seiner 2014 erschienenen Buchpublikation Breslau. Geschichte einer europäischen Metropole ${ }^{7}$ nimmt er die Vergangenheit der Oderstadt als eine Ikone der europäischen Kultur wahr, ungeachtet gewaltiger politischer und sozialer Umbrüche. Die Behauptung dieser Einzigartigkeit, die auch durch den vollständigen Bevölkerungsaustausch nach dem 2 . Weltkrieg nicht widerlegt werden könne, bekräftigt er im Vorwort:

${ }^{1}$ Kulak, Teresa: Wrocław-przewodnik historyczny. Wrocław 2000.

2 Davies, Norman, Moorhouse, Roger: Mikrokosmos. Portret miasta środkowoeuropejskiego. Wroctaw, Breslau, Vratislavia. Kraków 2002.

${ }^{3}$ Zawada, Andrzej: Bresław. Eseje o miejscach. Wrocław 1996.

${ }^{4}$ Schier, Roswitha: Literarischer Reiseführer Breslau - Sieben Spaziergänge. Potsdam 2004.

${ }^{5}$ Garber, Klaus: Das alte Breslau: Kulturgeschichte einer geistigen Metropole. Köln 2014.

${ }^{6}$ Ebd., s.: der Klappentext.

${ }^{7}$ Mühle, Eduard: Breslau. Geschichte einer europäischen Metropole. Köln Weimar Wien 2015. 
Dass diese Stadt 1945 materiell weitgehend zerstört und ihre Einwohnerschaft 1945-1948 nahezu komplett ausgetauscht worden ist, bedeutet keineswegs, dass diese stadtgeschichtliche Identität grundsätzlich in Zweifel gezogen werden muss - so sehr das persönliche Migrationsschicksal deutscher Alt-Breslauer und polnischer Neu-Breslauer dies in den Jahren von 1945 bis 1990 (und mitunter darüber hinaus) subjektiv auch nahegelegt haben mochte. ${ }^{8}$

Er unterstreicht, dass man im Thema Breslau „,von einem historischen Phänomen sprechen [kann], dessen Geschichte sich auch unter einem Namen - im vorliegenden deutschsprachigen Fall: Breslau - darstellen [lässt]".9

Bezüglich des Aufbaus ist die Monographie in zehn chronologische und vergleichbar umfangreiche Kapitel aufgeteilt. Die ersten drei beziehen sich auf das Mittelalter: „1. Frühmittelalterliche Burgstadt (950er-1230er Jahre)“, „2. Herzogliche Lokationsstadt (1230er-1330er Jahre)“ und „3. Patrizische Handelsmetropole (1330er-1520-er Jahre)“. Die weiteren zwei Abschnitte behandeln die Epoche der religiösen Auseinandersetzungen (,4. Hochburg des Luthertums, 1520er-1630er Jahre“, „5. Zielpunkt der Gegenreformation"). Anschließend bespricht Mühle die Zeiten der preußischen und deutschen Herrschaft: „6. Preußische Residenzstadt (1740er-1870er Jahre)“, „7. Regionales Zentrum der Moderne (1870er-1930er Jahre)“ wie auch „8. Bollwerk des Deutschen Ostens (1933-1945)““. Der polnischen Ära in der Stadtgeschichte wurden zwei abschließende Teile gewidmet. Der eine bespricht die Epoche des Kommunismus („9. Hauptstadt der Wiedergewonnenen Gebiete“), der letzte - die Zeit nach der Wende 1989 bis heute („10. Postsozialistische Großstadt"). Beachtenswert ist, dass der Autor auf die traditionelle Gliederung der Stadtgeschichte verzichtet. Im Gegensatz zum Standardwerk von Teresa Kulak, in dem die Aufteilung der Kapitel und somit Epochen durch die politische Zugehörigkeit der Stadt bestimmt wird („Unter den Piasten. Ca. 990-1335“, „Unter böhmischer Herrschaft“, „Unter der Herrschaft Habsburgs 1526-1740“, „Im preußischen Staat 1741-1945“ und: „Wrocław, die polnische Stadt" ${ }^{\prime 10}$ ), setzt Mühle auf andere Aspekte der Stadtgeschichte. In seiner Terminologie wird das Augenmerk nicht ausschließlich auf die politische Zugehörigkeit der Stadt gelegt, sondern auf die räumlich-funktionelle Entwicklung Breslaus und auf bedeutende religiöse, künstlerische oder soziale Prozesse, die die Odermetropole geprägt haben.

Um die Geschichte einer Großstadt auf 300 Seiten überschaubar, gut verständlich und trotzdem in einer dem Thema gerecht werdenden Komplexität zu schildern, entschied sich Eduard Mühle, ,die strukturellen Grundzüge und wichtigsten Entwicklungen [...] in großzügigem Überblick darzustellen und sie am Beispiel von epochensignifikanter Bauwerke und Persönlichkeiten [...] zu verdichten“. ${ }^{11}$ Jedes Kapitel wird durch die Beschreibung eines wichtigen Breslauer Baudenkmals eröffnet. In der Reihenfolge des Buches sind das: der romanische Dom (aus der Zeit der „frühmittelalterlichen Burgstadt"), der Ring (für die Zeit der „herzoglichen Lokationsstadt"), das Rathaus (als Wahrzeichen der ,patrizischen Handelsmetropole“), die Stadtbefestigung (für das Kapitel „Hochburg des Luthertums“), Jesuiten-Kolleg und alte Universität (als „Zielpunkt der Gegenreformation“), Hofkirche und Königsschloss (zum Kapitel „Preußische Residenzstadt“), die Jahrhunderthalle („Regionales Zentrum der Moderne“), das neue Regierungspräsidium (als Symbol für das

\footnotetext{
${ }^{8}$ Mühle (2015: 11).

${ }^{9}$ Mühle (2015: 12).

${ }^{10}$ Kulak (2006: 343).

11 Mühle (2015: 10).
} 
„Bollwerk des deutschen Ostens“), das Kościuszko-Wohnviertel (KDM, für den Abschnitt „Hauptstadt der Wiedergewonnenen Gebiete“) und als letztes, ein die „Postsozialistische Großstadt" bezeichnendes Gebäude, der Wolkenkratzer - Sky Tower. Eine Bereicherung der ganzen Publikation bilden farbige Fotografien, die das sich verändernde Gesicht der Odermetropole dokumentieren. Dass die Stadt - trotz großer Umbrüche in ihrer Geschichte - ein einzigartiges Phänomen ist, veranschaulichen passend zwei Bilder auf Seite 211: das von den Nationalsozialisten 1939-1945 erbaute neue Regierungspräsidium (heute das Wojewodschaftsamt) und das von den Kommunisten 1954-56 errichtete Kościuszko-Wohnviertel. Zwei von unterschiedlichen totalitären Regimen unter sehr unterschiedlichen politischen Verhältnissen erbaute Gebäude weisen in der Tat ähnliche architektonische Züge auf und dienten als Kulisse für Aufmärsche und Propagandaveranstaltungen. ${ }^{12}$

Nach der Darstellung eines wichtigen Bauwerks präsentiert der Autor bündig die Stadtgeschichte in der jeweiligen Epoche. Er erweitert sie um kurze Abschnitte zu ausgewählten Breslauer Persönlichkeiten. Durch solch eine Darstellungsweise ergänzt Mühle den geschichtlichen Kanon Breslaus um neue Figuren. So entschied sich der deutsche Historiker im Kapitel „2. Herzogliche Lokationsstadt (1230er-1330er Jahre)“ den Piastenherzog Heinrich IV. zu präsentierten, wohingegen Teresa Kulak in ihrer Geschichte Breslaus seinem berühmteren Großvater (Heinrich dem Frommen) und dem Urgroßvater (Heinrich dem Bärtigen) viel Platz einräumte. ${ }^{13}$

Auch für weitere Kapitel wurden ausgewählte Persönlichkeiten aus Breslau näher gebracht: Kaspar Popplau (,typischer Vertreter des Breslauer Patriziats“ im 15. Jahrhundert), Johannes Crato von Crafftheim - Humanist und angesehener Mediziner, Autor einer bahnbrechenden Abhandlung zur Pestbekämpfung - Ordnung oder Präservation zur Zeit der Pest (1555), der prominente Anwalt und Pazifist Adolf Heilberg (1858-1936), der wegen seiner politischen Weltanschauung aus Breslau nach dem Sieg der NSDAP 1933 fliehen musste, oder der Breslauer Historiker Hermann Aubin, der von Eduard Mühle als ein „durchschnittlicher“ Repräsentant der Mehrheit bezeichnet wurde, die das NS-Regime befürwortete: „ein anerkannter Wissenschaftler, ein Angehöriger der intellektuellen Elite, ein denkender und kluger Mann - und doch ein typischer Vertreter jener Mehrheit, die den Nationalsozialismus begrüßte, die sich ihm andiente und die Augen vor seinen Verbrechen verschloss, ja diese noch lange nach dem Untergang nicht wahrhaben wollte“ ${ }^{14} \mathrm{Zu}$ markanten Gestalten Breslaus zählt der Autor auch den für sein innovatives Pantomimentheater bekannten, polnischen Künstler Henryk Tomaszewski, der in Wrocław nach 1945 seine künstlerische Tätigkeit aufgenommen hat.

Mühles Bestreben, auch die allerneuste Geschichte der Stadt zu präsentieren, realisiert er in der treffenden und ausführlichen Analyse der neuen Investitions- und Revitalisierungswelle im polnischen Wrocław des 21. Jahrhunderts. Die viertgrößte Stadt Polens rühmt der Historiker, indem er ,,spektakuläre Neubauten [...], [die] enorm gestiegene Bedeutung Breslaus als Hochschul- und Wissenschaftsort [...], [den] enorme[n] Touristenzustrom [...] oder [das] erfolgreiche Bemühen um weitere Infrastrukturverbesserungen“" zur Sprache bringt. ${ }^{15}$ Jedoch taucht in diesem letzten Kapitel keine Biographie einer bedeutenden Persönlichkeit auf, weil der deutsche Historiker keinen Lebenden porträtieren wollte.

\footnotetext{
12 Vgl.: Mühle (2015: 243, 263).

13 Vgl.: Kulak (2006: 20-27).

${ }^{14}$ Mühle (2015: 251).

15 Mühle (2015: 313).
} 
Das Buch Breslau Geschichte einer europäischen Metropole von Eduard Mühle stellt eine neue Betrachtungsweise der Geschichte Breslaus dar. Dem Autor gelang es, eine überschaubare Geschichte Breslaus zu verfassen, in der er auf das Vergangene innovativ anhand von Beschreibungen der architektonischen Baudenkmäler und der bedeutenden historischen Gestalten eingeht. Hervorzuheben ist in diesem Sinne eine kreative Auswahl der präsentierten Breslauer Persönlichkeiten sowie eine erfolgreiche Wiedergabe der neusten Geschichte der Stadt nach 1945. Ein Novum sind auch die Kapitelnamen, die politische Zugehörigkeit der Stadt außer Acht lassen und sie als ein davon unabhängig funktionierendes Phänomen wahrnehmen. Es ist ein empfehlenswertes Buch für alle, die die Oderstadt als einzigartige europäische Kulturlandschaft entdecken wollen.

\section{Literatur}

Mühle, Eduard: Breslau. Geschichte einer europäischen Metropole. Köln Weimar Wien 2015. Kulak, Teresa: Wroctaw-przewodnik historyczny. Wrocław 2000.

Grzegorz Wilga

Bericht über die internationale Konferenz „Mehrsprachigkeit und Multikulturalität im translatorischen und glottodidaktischen Paradigma“, 9.-11.10.2015, Wrocław

DOI: $10.19195 / 0435-5865.141 .43$

Vom 9. bis 11. Oktober 2015 fand am Institut für Germanistik der Universität Wrocław eine internationale Konferenz zum Thema Mehrsprachigkeit und Multikulturalität im translatorischen und glottodidaktischen Paradigma statt, die von der Forschungsstelle für Translatorik und dem Lehrstuhl für Glottodidaktik veranstaltet wurde. Mit dem reich gefächerten Konferenzthema wollten die Organisatoren zu einer breiten Palette von interessanten und bereichernden Vorträgen und Diskussionen anregen.

Im Rahmen der Konferenz wurden sieben Sektionen angeboten, darunter die IATI$\mathrm{PhD}$-School, die dank der Zusammenarbeit der Forschungsstelle für Translatorik mit der International Academy for Translation and Interpreting (IATI) zustande kam, sowie ein Workshop. Die Sektionen waren folgenden Themenbereichen gewidmet:

Sprachen und Kulturen in Kontakt;

Rechtssprache;

Fremde Sprachen in Kontakt und Unterricht;

Literarisches Übersetzen;

Glottodidaktik;

Translatorik.

IATI-PhD-School. 